\title{
Bandwidth Enhancement of Super Wide Band Fractal Microstrip Patch Antenna for Wireless Applications
}

\author{
Abhinav Bhargava, Poonam Sinha
}

\begin{abstract}
In communication system the requirement of bandwidth enhancement is the most necessary requirement. This requirement of bandwidth increment can easily achieve the data rate of the system, so for further development of communication system higher bandwidth is required. Wireless communication already covered most of the frequency range till five giga hertz. In fractal technique structure which is similar in shape are designed for the similar nature of the radiation to increase the radiative power. By fractal radiation area can be increased, is used to achieve the bandwidth of the signal. By fractal technique size of the antenna can also be reduced for miniaturization of the device which is going to be developed. In this paper a different rectangular antenna is presented, consists of dual properties, is fractal and defected ground. This paper shows simulated result and designed hardware result and also shows the comparative result of these. In defective one rectangular slot has been introduced. A higher bandwidth rectangular antenna has been simulated and designed and a comparative analysis of simulated and hardware results compared and analyzed. Simulation done by considering $4 \mathrm{GHz}$ as center frequency. The easily available material FR-4 is used for simulation as well as hardware design. In this substrate thickness will be 1.6mm. Return loss of the given design is achieved less than $-10 \mathrm{db}$ for almost $11 \mathrm{GHz}$. In future the many applications will use this range of frequency. The value of VSWR is also achieved less than two for $3 \mathrm{GHz}$ to $14 \mathrm{GHz}$. Simulation of this design is done by HFSS software. The ground plane is also modified for enhancement of bandwidth.
\end{abstract}

Keywords : Enhanced Bandwidth, fractal patch-antenna, Defected Ground, return loss, VSWR, radiation pattern.

\section{INTRODUCTION}

$\mathrm{H}_{\mathrm{e}}$ fractal and different fractal geometries are explained. The detail of HFSS simulation software tool is used for analysis and study. In the beginning term „,fractal "e was invented by the mathematician B.B. Mandelbrot, he belongs from France, invent fractal in 1970. A fractal is discontinuous structure

Revised Manuscript Received on February 05, 2020.

* Correspondence Author

Abhinav Bhargava*, PhD Scholar, Department of Electronics \& Communication Engineering, Barkatullah University Institute of Technology, Bhopal, India, abhina.bhargava@gmail.com

Poonam Sinha, Head, Department of Electronics \& Communication Engineering, Barkatullah University Institute of Technology, Bhopal, India, poonamuit@yahoo.com

(C) The Authors. Published by Blue Eyes Intelligence Engineering and Sciences Publication (BEIESP). This is an open access article under the CC BY-NC-ND license (http://creativecommons.org/licenses/by-nc-nd/4.0/) which is split into multiple parts in which increase the current length and size reduction can also be done by fractal technique.. When single shape is repeated multiple times called iteration produces fractal antenna. This iteration can go on without break multiple times can be infinitely, but practically infinite is not possible so huge length and boundary is developed. In now days in many field of science and engineering fractal term is used. Antenna is the field in which fractal term is used for bandwidth enhancement. For many communication system antenna are used and development is going on day by day. Many features can be developed and improved by fractal technique; size deduction and bandwidth enhancement are of them. The most common and important property of fractal are similarity and filling of space. The similarity produced the increment in number of resonant bands in the antenna called multiband antenna. By fractal the super wide band antenna can be designed and developed; which is used for wide band transmitter and receiver. Because of multiband behavior of the fractal. By fractal and its other properties size reduction is very easy, some example are Hilbert curve, Sierpinski Gasket etc.[4] Some of the famous fractal technique is called sierpinski gasket shows repetitive triangle inside the triangle. So many other shapes can also be used for designing of fractal antenna. When one of geometry introduced inside the other again and again these are called iterations, by these iteration size reduction and band. The repetitive fractal shape reduces size of antenna. In the field of wireless communication many communication systems have to design with different resonant frequency for this need of different antenna is there. So the solution of this problem is to use multiple antennas for single device or use a wideband antenna. The first solution is costly and complex. Size reduction is also a huge challenge in the antenna designing which can be achieved with fractal optimization. Some other technique is also available like defected ground and thickness of dielectric material etc.

\section{PRE EXISTING GEOMETRY}

As shown in the figure 1 the swastika structure is given, which shows three different geometries, first geometry gives single swastika on full PCB. This design is further modified in the second geometry called stage 1 . In stage one four extra swastika has been added called fractal. In the stage 2 again four swastikas added to achieve the similarity property of the antenna and get enhance bandwidth. By these iterations electric length increment as well as bandwidth can be achieved. 
But this type complex design can't be easily implemented on the hardware. The life of this type of complex geometry is not very long so required to design an antenna with the simple structure.

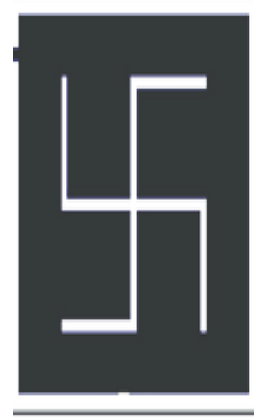

Stage 0

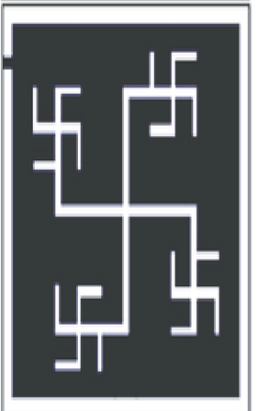

Stage l

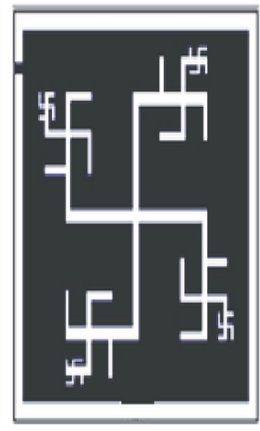

Stage2
Figure 1: Existing Designs-1

So basic rectangular geometry is easy to design and implement. This paper shows the basic rectangular slotted rectangular antenna. Another pre existing geometry is represented in the figure number two.

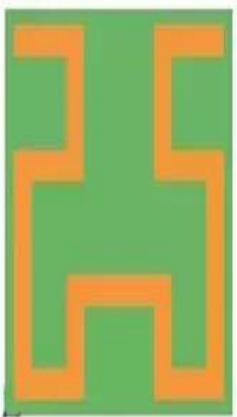

Iteration-1

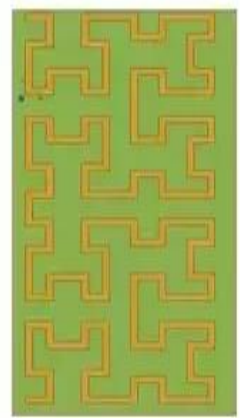

Iteration-2

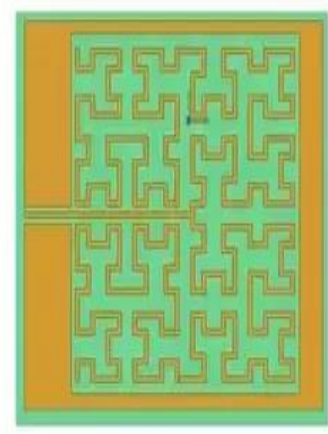

Iteration-3
Figure 2: Existing Designs-2

Figure number two shows another example of fractal geometry with three iterations in this first geometry shows the chain of rectangle which further enlarged in the second and third iteration. But the problem with this geometry is complexity. This is shorted by simple geometry presented in this paper.

\section{PROPOSED DESIGNE GEOMETRY}

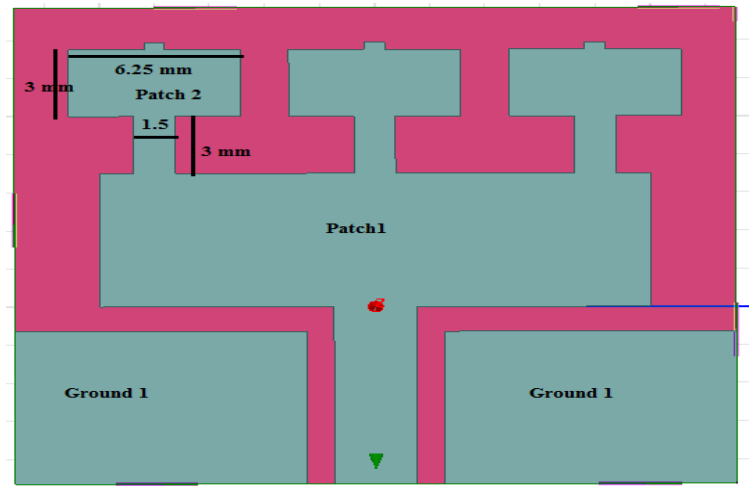

Figure 3: Front view of proposed antenna

Figure number three shows the proposed antenna with multi rectangle and modified ground plane. By this simple design enhanced electrical length has been achieved. A super wideband antenna has been simulated and designed. One notch is also used for the wideband antenna design.

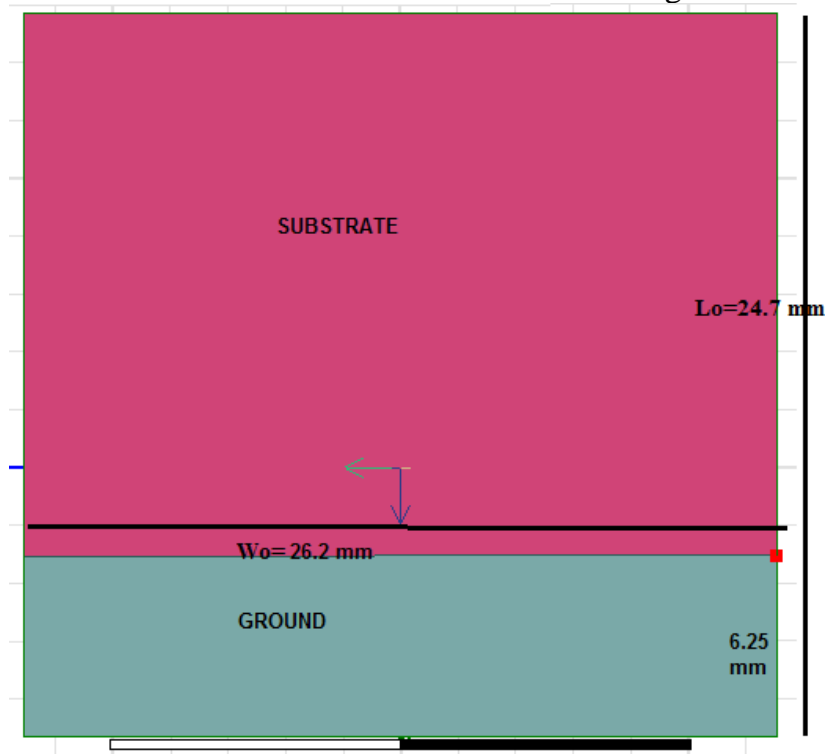

Figure 4: Back View of Proposed compact super wideband antenna

Back side view of the proposed design is given with modified structure. In this in place of full ground plane a modified $26.2 * 6.25 \mathrm{~mm}$ ground plane is given.

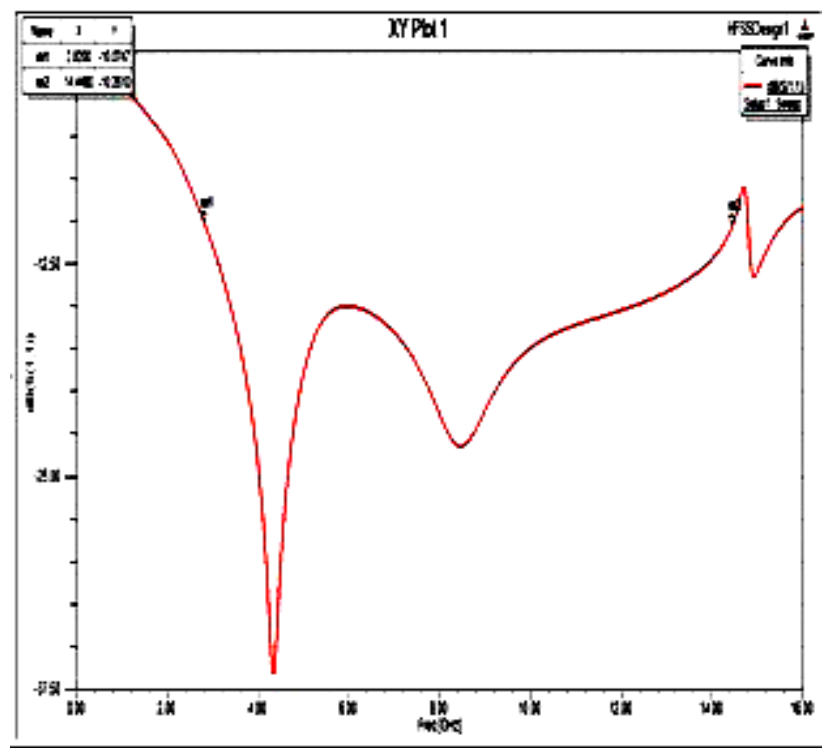

Figure 5: S11Versus Frequency Plot of super wideband Antenna

Figure number five demonstrate the VSWR for proposed design in which value of VSWR is achieved less than two for $3 \mathrm{GHz}$ to $14 \mathrm{GHz}$.

\section{HARDWARE DESIGN}

As given in figure 6 the hardware is demonstrated which consist of one SMA connector is connected with the patch and this antenna is fabricated by FR-4 substrate in this the value of permittivity is 4.4 .

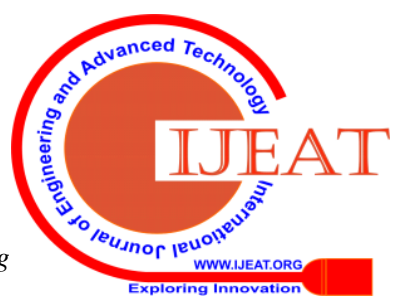




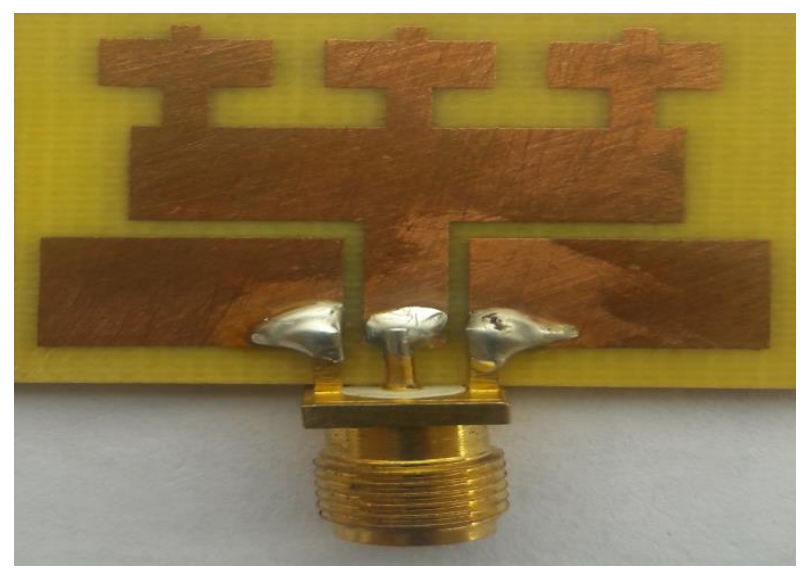

Figure 6: Front View of Hardware of Proposed compact super wideband antenna

The measured value of the proposed design is given in the figure 7 shows the bandwidth of $9.7 \mathrm{GHz}$ is achieved from $1.7 \mathrm{GHz}$ to $11.4 \mathrm{GHz}$. There is difference of $1.3 \mathrm{GHz}$ is observed in the analysis due to the quality of the PCB and connector loss etc.

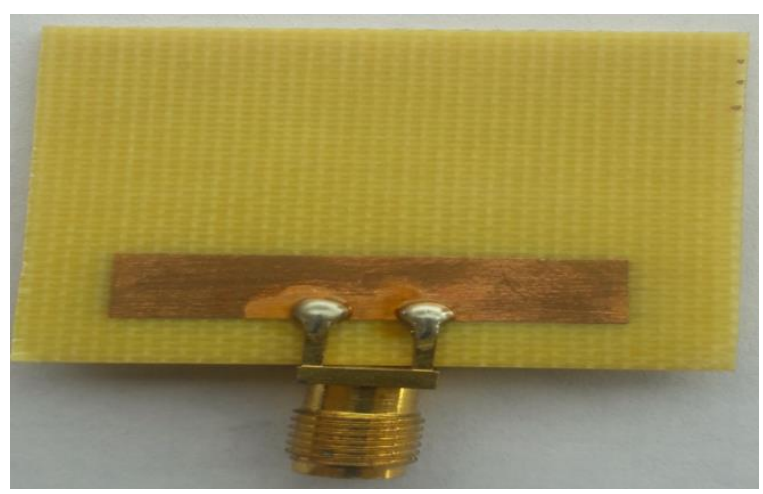

Figure 7: Back View of Hardware of Proposed compact super wideband antenna

Figure number seven shows the back side view of the proposed antenna design hardware.

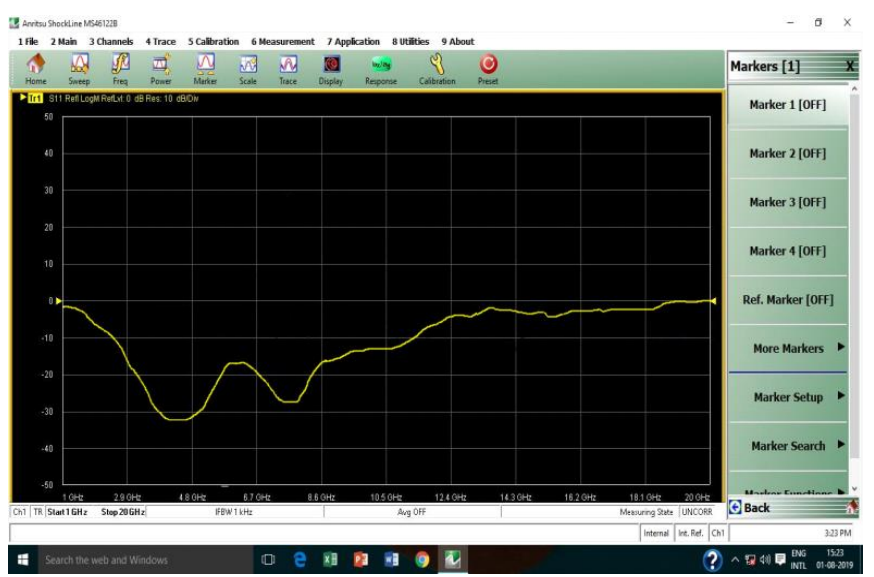

Figure 8: Shows the VSWR of Hardware of Proposed compact super wideband antenna
Table 1: Comparison of Simulation and hardware tested results

\begin{tabular}{|c|c|c|c|c|c|c|}
\hline $\begin{array}{l}\text { Resonating } \\
\text { Frequency } \\
\text { (GHz) }\end{array}$ & $\begin{array}{l}\text { Return } \\
\text { Loss } S_{11} \\
\text { (dB) }\end{array}$ & \begin{tabular}{|l|} 
Return \\
Loss $\quad S_{11}$ \\
Hardware \\
(dB)
\end{tabular} & VSWR & Directivity & $\begin{array}{l}\text { Bandwidth } \\
\text { (GHz) }\end{array}$ & $\begin{array}{l}\text { Bandwidth of } \\
\text { Hardware } \\
\text { (GHz) }\end{array}$ \\
\hline $4 \mathrm{GHz}$ & $-37.5 \mathrm{~dB}$ & $-32 \mathrm{~dB}$ & 1.1 & $3.3 \mathrm{dBi}$ & 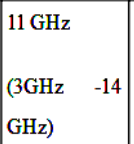 & 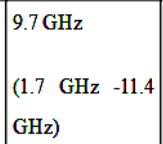 \\
\hline
\end{tabular}

\section{CONCLUSION}

An antenna of using fractal technique is simulated and designed. The comparative analysis is also observed in which the value of bandwidth is differ by $1.3 \mathrm{GHz}$. This difference occurs due to some limitation of printed circuit board and connector error. By simulation $11 \mathrm{GHz}$ bandwidth is achieved on the other end by hardware $9.7 \mathrm{GHz}$ bandwidth is measured. This following information is also given in the comparison table 1.

\section{REFERENCES}

1. GAkankshaFarswan; Anil Kumar Gautam; Binod Kumar Kanaujia; KarumudiRambabu "Design of Koch Fractal Circularly Polarized Antenna for Handheld UHF RFID Reader Applications" IEEE Transactions on Antennas and PropagationVol. 64, pp. 771-775, 2016.

2. IraklisGiannakis; Antonios Giannopoulos; Craig Warren "A Realistic FDTD Numerical Modeling Framework of Ground Penetrating Radar for Landmine Detection"IEEE Journal of Selected Topics in Applied Earth Observations and Remote SensingVol. 9, pp. 37-51, 2016.

3. M. Kgwadi; T. D. Drysdale "Diode-switched thermal-transfer printed antenna on flexible substrate" Vol. 52, pp. 258-260, 2016.

4. JayaramKizhekkePakkathillam; MalathiKanagasabai“Circularly Polarized Broadband Antenna Deploying Fractal Slot Geometry" IEEE Antennas and Wireless Propagation Letters Vol. 14, pp. 1286-1289, 2015.

5. Yongjiu Li; Long Li; Yongliang Zhang; Chunsheng Zhao“Design and Synthesis of Multilayer Frequency Selective Surface Based on Antenna-Filter-Antenna Using Minkowski Fractal Structures” IEEE Transactions on Antennas and PropagationVol. 63, pp. 133-141, 2015.

6. SayantanDhar; Kaushik Patra; RowdraGhatak; Bhaskar Gupta; Dipak RanjanPoddar"A Dielectric Resonator-Loaded Minkowski Fractal-Shaped Slot Loop Heptaband AntennaIEEE Transactions on Antennas and PropagationVol. 63, pp. 1521-1529, 2015.

7. TomislavDebogovic and Julien Perruisseau-Carrier "Array fed partially reflective surface antenna with independent scanning and beamwidth dynamic control” IEEE Trans. Antennas Propag., vol. 62, no. 1,pp. 446-449, Jan. 2014.

8. VoryaWaladi, NooshinMohammadi, YasharZehforoosh, AsiehHabashi, and JavadNourinia "A novel modified star-triangular fractal(MSTF) monopole antenna for super-wideband application," IEEE Antennas and Wireless Propagation Lett. Vol. 12 pp. 651-654, 2013.

9. Jian Li; Tianyan Jiang; Changkui Cheng; Caisheng Wang "Hilbert fractal antenna for UHF detection of partial discharges in transformers" IEEE Transactions on Dielectrics and Electrical Insulation Vol. 20, pp. 2017-2025, 2013.

10. AbolfazlAzari; AlyaniIsmail; AduwatiSali; FazirulhisyamHashim"A New Super Wideband Fractal Monopole-Dielectric Resonator Antenna” IEEE Antennas and Wireless Propagation Letters Vol. 12, pp. 2014-2016, 2013

11. Abhinav Bhargava; Poonam Sinha; "Design and Simulation of Rectangular Micro-strip Dual Band Single Slot Patch Antenna" Vol. 72, pp. 12-15, 2013. 
12. Abhinav Bhargava; Anshul Shrivastava "A Comparative Study of Different Shaped Patch Antennas with and Without Slots" Vol. 2, pp. 3306-3308, 2014.

13. Abhinav Bhargava; Anshul Shrivastava "A Multiple U Slotted Rectangular Micro-strip Patch Antenna” Vol. 1, pp. 1-3, 2012.

14. Abhinav Bhargava; Shwati Shrivastava "Design \& Simulation of Triple Band Micro Strip Patch Antenna for FPV, Wi-Max and W-Lan Application" Vol. 2, pp. 7-10, 2015.

\section{AUTHORS PROFILE}

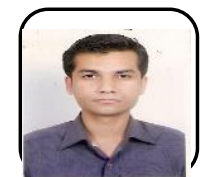

Abhinav Bhargava, pursuing $\mathrm{PhD}$ in Electronics \& Communication Engineering from Barkatullah University Institute of Technology, Bhopal, India. He had received M.Tech (DC) degree from the same University in 2012. His research interest is antenna designing.

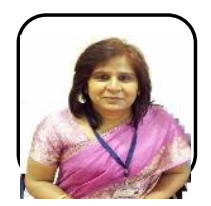

Dr. Poonam Sinha is working as Head in the Department of Electronics \& Communication Engineering at Barkatullah University Institute of Technology, Bhopal, India. She has received PhD from MANIT, Bhopal, India. Her research interest area is image processing, Antenna designing etc. She has published many papers in International and National conference and journals. 